Laboratories. The near patient tests and ELISAs were provided

by SmithKline Diagnostics.

Competing interests: None declared.

1 Office of Health Economics. Compendium of health statistics. 10th ed. London: Office of Health Economics, 1997.

2 British Society of Gastroenterology. Dyspepsia management guidelines [abstract]. London: British Society of Gastroenterology, 1996.

3 American Gastroenterological Association. American Gastroenterological Association medical position statement: evaluation of dyspepsia. Gastroenterology 1998;114:579-81.

4 Stone M, Mayberry J, Wicks A, Livsey S, Stevens M. The Helisal test: an assessment of readability and performance [abstract]. Gut 1996;39:110.

5 Peitz U, Tillenburg B, Baumann M. Insufficient validity of a new rapid whole blood test for Helicobacter pylori (HP) infection [abstract]. Gastroenterology 1996;110:226.

6 Moayyedi P, Carter A, Catto A, Heppell R, Grant P, Axon A. Validation of a rapid whole blood test for diagnosing Helicobacter pylori. BMJ 1997;314:119.
7 Reilly T, Poxon V, Sanders D, Elliott T, Walt R. Comparison of serum, salivary, and rapid whole blood diagnostic test for Helicobacter pylori and their validation against endoscopy tests. Gut 1997;40:454-8.

8 Kroser J, Faigel D, Furth E, Metz D. Comparison of rapid office-based serology with formal laboratory-based ELISA testing for diagnosis of Helicobacter pylori gastritis. Dig Dis Sci 1998;43:103-8.

9 Jones R, Phillips I, Felix G, Tait C. An evaluation of near-patient testing for Helicobacter pylori in general practice. Aliment Pharmacol Ther 1997;11: $01-5$

10 Talley N, Lambert JR, Howell S, Lin HHX, Agreus L. An evaluation of whole blood testing for Helicobacter pylori in general practice. Aliment Pharmacol Ther 1998;12: 641-5.

11 Duggan A, Logan RPH, Knifton A, Logan RFA. Accuracy of near-patient blood tests for Helicobacter pylori. Lancet 1996;348:617.

12 Churchill RD, Hill PG, Holmes GKT. Breath test is better than near patient blood tests. BMJ 1998;316:1389.

(Accepted 19 July 1999)

\title{
Antibiotic prescribing and antibiotic resistance in community practice: retrospective study, 1996-8
}

\author{
J T Magee, Emma L Pritchard, Karen A Fitzgerald, F D J Dunstan, A J Howard on behalf of the \\ Welsh Antibiotic Study Group
}

We describe a retrospective survey of antibiotic prescribing in general practitioners' surgeries and resistance to antibiotics in Wales from March 1996 to April 1998.

\section{Methods and results}

Data on the susceptibility to antibiotics of coliform organisms in routine urine samples taken by general practitioners for diagnosis of urinary tract infections were collected from the Bangor, Cardiff, and Rhyl Public Health Laboratories and the East Glamorgan, Prince Charles, and Wrexham Maelor Hospitals. Data on the prescribing practices of surgeries were obtained from the Welsh Prescription Pricing Service. Rates of prescribing (the number of prescriptions/1000 patients per year) and resistance rates (which excluded multiple isolates of organisms with the same susceptibility from the same patient) were calculated for each surgery. The use of broad spectrum penicillin formulations without a $\beta$ lactamase inhibitor (such as ampicillin and amoxicillin) was estimated by subtracting the number of prescriptions for co-amoxiclav from the total number of prescriptions for all other broad spectrum penicillins. We use the term amoxicillin below to refer to these broad spectrum penicillins without a $\beta$ lactamase inhibitor.

Resistance rates for surgeries which were based on fewer than 50 isolates were excluded, leaving data on about 30000 isolates from 190 general practitioner surgeries serving about 1200000 patients. We sought to identify the effects of bias caused by the selective submission of urine samples by examining the relation between resistance rates and sampling (number of urine specimens/1000 registered patients) and the relation between positivity (number of coliform isolates/100 samples or 1000 registered patients) and prescribing or sampling.

The use of antibiotics and rates of resistance to antibiotics varied between surgeries; the correlation between the prescribing of an antibiotic and resistance to the same antibiotic was often significant (table). The correlation was also significant between the use of amoxicillin and resistance to trimethoprim and vice versa. Combined resistance to ampicillin and trimethoprim occurred in 21\% (6782/32 532) of isolates and was significantly associated with the use of both trimethoprim and amoxicillin $(\mathrm{P}<0.001)$. The correlation between the use of amoxicillin and resistance to trimethoprim and vice versa was lost when strains exhibiting combined resistance to both agents were removed from the analysis.

There was no significant correlation between antibiotic use and the number of urine specimens submitted for testing per 1000 registered patients or the number of coliform isolates in urine samples per 1000 registered patients. The number of isolates per 1000 registered patients correlated linearly with the number of urine specimens submitted per 1000 registered patients $\left(\mathrm{P}=0.001, r_{\mathrm{s}}=0.9585\right)$.

\section{Comment}

The results show that there is a correlation between antibiotic resistance in coliform organisms in urine samples and the use of antibiotics by a general practice. This is the first survey to suggest that geographically localised effects from antibiotic use occur in communities.

The dynamics of the emergence, spread, and maintenance of antibiotic resistance in populations are still unclear. ${ }^{1}$ Much of the prescribing described here is likely to have been related to treatment of respiratory infections, and this may have been an important factor in determining the observed resistance. Resistance could be occurring through the prior selection of antibiotic resistant coliform organisms in the faecal flora ${ }^{2}$ of patients presenting with urinary infections or by transmission of such organisms by others in the community.

Coselection of resistance to trimethoprim and ampicillin is explainable. Transmissible plasmids that code for combined resistance to ampicillin and trimethoprim are common in Escherichia coli ${ }^{3}$; there-
Department of Medical

Microbiology and

Public Health

Laboratory,

University Hospital

of Wales, Cardiff

CF14 4XW

J T Magee

clinical scientist

A J Howard

group director, $P H L S$

Wales

University of Wales College of

Medicine, Cardiff

CF14 4XW

Emma L Pritchard medical student

Bro Taf Health

Authority, Cardiff

CF1 4TW

Karen A Fitzgerald

pharmaceutical

adviser

Department of

Medical Computing and Statistics,

University of Wales

College of Medicine

F D J Dunstan

senior lecturer

Correspondence to:

A J Howard

tony.howard@

phls.wales.nhs.uk

BMJ 1999;319:1239-40

website

extra

A complete list of the members of

the study group

appears on the

BMJ's website

www.bmj.com 
$\mathrm{P}$ value and Spearman's correlation coefficient (95\% confidence interval) for use of antibiotics and rate of antibiotic resistance (excluding repeat isolates) among general practitioner surgeries in Wales, 1996-8

\begin{tabular}{|c|c|c|c|c|c|c|c|c|c|c|c|c|}
\hline \multirow[b]{2}{*}{ Antibiotic used } & \multicolumn{2}{|c|}{ Ampicillin } & \multicolumn{2}{|c|}{ Co-amoxiclav } & \multicolumn{2}{|c|}{ Cephalosporin* } & \multicolumn{2}{|c|}{ Trimethoprim } & \multicolumn{2}{|c|}{ Ciprofloxacin } & \multirow{2}{*}{$\begin{array}{l}\text { Mean (range) } \\
\text { No of } \\
\text { prescriptions/ } \\
1000 \\
\text { patients/year }\end{array}$} & \multirow{2}{*}{$\begin{array}{l}\text { Mean (range) } \\
\text { percentage of } \\
\text { organisms } \\
\text { resistant to } \\
\text { antibioticst }\end{array}$} \\
\hline & $P$ value & $r_{\mathrm{s}}$ & $P$ value & $r_{\mathrm{s}}$ & $P$ value & $r_{\mathrm{s}}$ & $P$ value & $r_{\mathrm{s}}$ & $P$ value & $r_{s}$ & & \\
\hline Amoxicillin & $<0.001$ & $\begin{array}{c}0.394(0.269 \\
\text { to } 0.506)\end{array}$ & 0.659 & & 0.527 & & 0.015 & $\begin{array}{c}0.172 \\
(0.033 \text { to } \\
0.304)\end{array}$ & 0.376 & & $352(81-912)$ & $53.2(34.2-67.6)$ \\
\hline Co-amoxiclav & 0.576 & & 0.714 & & 0.145 & & 0.936 & & 0.309 & & 75 (7-239) & $8.4(0.0-26.1)$ \\
\hline Cephalosporin & 0.108 & & 0.753 & & 0.018 & $\begin{array}{c}0.167 \\
(0.028 \text { to } \\
0.299)\end{array}$ & 0.134 & & 0.478 & & 107 (16-532) & $6.5(0.0-19.0)$ \\
\hline Trimethoprim & 0.004 & $\begin{array}{c}0.204(0.066 \\
\text { to } 0.334)\end{array}$ & 0.003 & $\begin{array}{c}0.229 \\
(0.081 \text { to } \\
0.367)\end{array}$ & 0.001 & $\begin{array}{c}0.230 \\
(0.094 \text { to } \\
0.358)\end{array}$ & $<0.001$ & $\begin{array}{c}0.331 \\
(0.201 \text { to } \\
0.450)\end{array}$ & 0.056 & & $63(13-215)$ & $26.3(12.7-44.3)$ \\
\hline Quinolones & 0.069 & & 0.873 & & 0.103 & & 0.306 & & 0.015 & $\begin{array}{c}0.225 \\
(0.045 \text { to } \\
0.391)\end{array}$ & $34(7-96)$ & $2.2(0.0-10.1)$ \\
\hline All antibiotics & $<0.001$ & $\begin{array}{c}0.319(0.188 \\
\text { to } 0.439)\end{array}$ & 0.581 & & 0.411 & & 0.031 & $\begin{array}{c}0.153 \\
(0.014 \text { to } \\
0.286)\end{array}$ & 0.141 & & 971 (400- 1739) & \\
\hline
\end{tabular}

${ }^{*}$ Resistance to cefalexin was identified at six laboratories and resistance to cefradine at one.

†Overall mean of resistance rates for individual surgeries based on number of isolates varying from 50 to about 800 .

fore, selection pressure for resistance to one of these antibiotics is likely to select for resistance to the other.

The association between prescribing and resistance could have been caused by sampling bias if practices that had high rates of prescribing antibiotics were more selective in submitting samples for analysis, reserving testing for cases of treatment failure or complicated urological problems. In such cases it is likely that increased resistance would have been associated with lower rates of sampling. This was not observed. Few of the species of the coliform isolates were identified. Most were probably Escherichia coli, and it is unlikely that the small proportion of other coliform organisms (which often show broader resistance to antibiotics) would vary significantly between practices and be associated only with surgeries with high rates of prescribing. Other confounding variables may explain the observed correlations but it seems probable that the relations reflect a causal Darwinian association between prescribing and resistance.

These findings bring the debate on prescribing in the community from the national to the local level and provide preliminary evidence that practitioners may have to face the broader consequences of their antibiotic prescribing among their own patients.
We thank the staff of the laboratories involved; the staff of the Prescription Pricing Authority; Drs Hefin Jones and Idris Humphreys, chairmen of the local medical committees, who gave permission for us to access the prescribing data; David Myles, University of Wales College of Medicine, for computing support; and David Livermore for his constructive comments.

Contributors: AJH conceived the original idea for the study, obtained approval for the collection of data, critically edited the paper, and gave final approval for it. AJH is guarantor for the study. JTM compiled and analysed the data, programmed the analysis macros, and drafted and edited the paper. ELP performed the pilot study, providing the impetus to proceed, and manually processed data that were invaluable in checking the validity of programs. KAF obtained the prescribing data and provided pharmaceutical advice. FDJD provided statistical guidance and checked the results. ELP, KAF, and FDJD participated in editing the paper.

Funding: Public Health Laboratory Service.

Competing interests: None declared.

1 Standing Medical Advisory Committee, Sub-Group on Antimicrobia Resistance. The path of least resistance. London: Stationery Office, 1998.

2 London N, Nijsten R, Mertens P, van der Bogaard A, Sotobberingh E. Effect of antibiotic therapy on the antibiotic resistance of faecal Escherichia coli in patients attending general practitioners. J Antimicrob Chemother 1994;34:239-46.

3 Amyes SGB. The success of plasmid-encoded resistance genes in clinical bacteria-an examination of plasmid-mediated ampicillin and trimethoprim resistance genes and their resistance mechanisms. J Med Microbiol 1989;28:73-83.

(Accepted 19 July 1999)

\section{Email submissions from outside the United Kingdom}

We are now offering an email submission service for authors from outside the UK. The address is papers@bmj.com

Ideally our email server would link seamlessly with our manuscript tracking system, but for now it does not, which is why we are offering the service only to authors outside the UK Most post in the UK arrives the next day, so UK authors have the least to gain in speed of delivery from email delivery. As soon as our systems improve we will invite email submissions from everyone.

If you choose to send your submission by email please would you send the text and any tables and figures as attached files, together with a covering letter giving all your contact details (postal address, phone, fax, and email address). We can read files created with most word processing, graphics, and spreadsheet programs.

When your submission is received in our email box you will receive an automatic acknowledgment to show that it has arrived. If the submission is incomplete we will contact you and ask you to resend the missing information.

Once the submission is complete we will register it on our manuscript tracking system and you will receive a standard acknowledgment in the post.

Letters to the editor should continue to be sent direct to www.bmj.com as rapid responses or to letters@bmj.com

papers@bmj.com 